10

\title{
Излучательная эффективность терагерцовых антенн с традиционной топологией и металлической метаповерхностью: сравнительный анализ
}

\author{
(ㅇ Д.В. Лаврухин ${ }^{1,2}$, А.Э. Ячменев ${ }^{1}$, И.А. Глинский ${ }^{1}$, Н.В. Зенченко ${ }^{1,2}$, Р.А. Хабибуллин ${ }^{1}$, \\ Ю.Г. Гончаров ${ }^{3}$, И.Е. Спектор ${ }^{3}$, К.И. Зайцев ${ }^{2,3}$, Д.С. Пономарев ${ }^{1}$
}

${ }^{1}$ Институт сверхвысокочастотной полупроводниковой электроники имени В.Г. Мокерова РАН, 117105 Москва, Россия

${ }^{2}$ Московский государственный технический университет имени Н.Э. Баумана, 105005 Москва, Россия

${ }^{3}$ Институт общей физики им. А.М. Прохорова РАН, 119991 Москва, Россия

e-mail: denis_lavruhin@mail.ru

Поступила в редакцию 17.12.2019 г.

В окончательной редакции 10.01.2020 г.

Принята к публикации 28.02.2020 г.

Экспериментально исследованы характеристики фотопроводящих антенн (ФПА) - источников излучения с традиционной топологией и с металлической метаповерхностью в виде плазмонных решеток, изготовленных на основе сверхрешеточных гетероструктур InGaAs/InAlAs. Измерены фототоки и спектры генерации образцов ФПА, определены энергетические характеристики терагерцового (THz) излучения и эффективность оптико-THz преобразования энергии лазерного излучения в электромагнитные колебания $\mathrm{THz}$ диапазона при различном напряжении смещения и средней мощности лазерного возбуждения. Для ФПА с метаповерхностью экспериментально продемонстрированы интегральная мощность ТНz излучения $10 \mu \mathrm{W}$ и эффективность преобразования до $0.2 \%$, недостижимые для антенн с традиционной топологией из-за их теплового пробоя. Таким образом, можно утверждать, что ФПА с метаповерхностью являются эффективными источниками $\mathrm{THz}$ излучения и могут стать элементной базой для построения систем $\mathrm{THz}$ спектроскопии, связанных с решением задач медицинской диагностики.

Ключевые слова: терагерцовая импульсная спектроскопия, элементная база терагерцовой оптотехники, фотопроводящая антенна, плазмонная решетка, метаповерхность, плазмонная локализация оптического поля, полупроводники, неинвазивная медицина.

DOI: $10.21883 /$ OS.2020.07.49575.19-20

\section{Введение}

Применение методов терагерцовой $(\mathrm{THz})$ импульсной спектроскопии и визуализации для решения прикладных задач медицинской диагностики злокачественных новообразований различной нозологии и локализации в последнее время приобретает все большую актуальность [1-4]. В качестве достижений можно выделить возможности применения $\mathrm{THz}$ технологий в задачах ранней неинвазивной и интраоперационной диагностики злокачественных новообразований кожи и слизистой [5-9], минимально-инвазивной и интраоперационной диагностики новообразований кишечника $[10,11]$ и желудка [12], а также интраоперационной диагностики новообразований молочной железы [13-15] и головного мозга [16-19]. Благодаря возможности получать одновременно амплитудную и фазовую информацию об отраженных или прошедших через объект исследований электромагнитных волнах когерентные (импульсные) методы генерации и детектирования $\mathrm{THz}$ излучения являются наиболее перспективным подходом к решению задач медицинской диагностики $[3,20]$. Такие системы эффективно работают при комнатной температуре, обеспечивая динамический диапазон вплоть до $110 \mathrm{~dB}$ [21] в полосе частот от 0.1 до $4.5 \mathrm{THz}$.

Наиболее распространенными источниками и детекторами излучения в импульсных $\mathrm{THz}$ системах являются фотопроводящие антенны (ФПА) на основе GaAs и его тройных соединений. Следует отметить, что довольно большое значение ширины запрещенной зоны в GaAs $\left(E_{g}=1.42 \mathrm{eV}\right)$ подразумевает использование сложной и громоздкой оптической системы с накачкой видимым излучением Ti:Sapphire-лазера с центральной длиной волны $800 \mathrm{~nm}$. При этом твердые растворы $\operatorname{In}_{x} \mathrm{Ga}_{1-x} \mathrm{As}$ позволяют работать (в зависимости от мольной доли индия $x$ ) с ИК лазерной накачкой в диапазоне длин волн $1.03-1.56 \mu \mathrm{m}$, излучаемой компактными и недорогими волоконными лазерными системами. К сожалению, слои InGaAs имеют довольно низкое электрическое сопротивление и большие времена жизни фотовозбужденных носителей заряда, что сильно затрудняет их широкое использование в системах $\mathrm{THz}$ спектроскопии и, в частности, в ФПА на их основе. Авторами многих зарубежных 
работ предпринимались различные попытки по улучшению указанных характеристик InGaAs, например, путем ионной имплантации [22,23] или низкотемпературного роста с компенсирующим легированием [24-26]. Однако среди прочих подходов наиболее успешным оказался эпитаксиальный рост фотопроводящих сверхрешеточных гетероструктур (ФСГ) InGaAs/InAlAs, которые в зависимости от применения можно разделить на три группы.

1. ФСГ InGaAs/InAlAs, легированные атомами эрбия. Такие структуры имеют сверхмалые времена жизни фотовозбужденных носителей заряда при относительно высокой их подвижности, и поэтому могут использоваться как в ФПА-источниках излучения, так и ФПАдетекторах [27].

2. ФСГ InGaAs/InAlAs, выращенные при пониженной температуре с дополнительным легированием фотопроводящих слоев InGaAs атомами бериллия. Такие структуры имеют сверхмалые времена жизни фотовозбужденных носителей и отлично подходят для использования в ФПА-детекторах [26].

3. Решеточно-согласованные и решеточно-напряженные нелегированные ФСГ InGaAs/InAlAs с барьерными слоями InAlAs, выращенными при пониженной температуре. Фотовозбужденные носители в таких структурах имеют довольно высокую подвижность и относительно малое временя жизни. Такие структуры используются в ФПА-источниках излучения [28,29].

Важно отметить, что динамический диапазон импульсных систем $\mathrm{THz}$ спектроскопии в значительной степени определяется параметрами ФПА-источника излучения. Поскольку компактные волоконные лазеры для возбуждения ФПА обладают средней мощностью ИК излучения $\sim 10 \mathrm{~mW}$, это накладывает принципиальное ограничение на минимальную эффективность оптико$\mathrm{THz}$ преобразования энергии в ФПА [30,31]. На сегодняшний день наиболее успешным методом повышения эффективности преобразования является формирование диэлектрической или металлической метаповерхности в зазоре ФПА [32]. В частности, метаповерхности в виде металлических плазмонных электродов заметно усиливают локализацию лазерного излучения накачки в области контакта металла решетки с фотопроводящим слоем и соответственно увеличивают эффективное поглощение лазерного излучения в фотопроводящем слое [33]. Показано, что эффективность решетки растет пропорционально ее аспектному соотношению другими словами, отношению высоты единичного плазмонного электрода к периоду решетки [34-36]. Технологические ограничения на максимальную толщину слоя электронного резиста, а также необходимость при экспонировании электронного резиста компенсировать эффекты, связанные с близостью соседних электродов, приводят к тому, что чем больше аспектное соотношение, тем дороже и сложнее в изготовлении становится решетка.
Еще один путь повышения эффективности связан с подбором энергии фотонов накачки ФПА. Известно, что при накачке узкозонных ФСГ лазерным излучением видимого диапазона $(780-800 \mathrm{~nm})$ фотовозбужденные носители заряда („горячие“ носители) испытывают сильное междолинное рассеяние, и их времена жизни несколько увеличиваются [37,38]. Однако показано, что в этом случае больший коэффициент поглощения усиливает локализацию энергии оптического импульса вблизи поверхности фотопроводящего слоя/электродов. Другими словами, в ряде случаев [38,39] бо́льшая энергия фотонов может обеспечить бо́льшую интегральную мощность ТГц излучения по сравнению с возбуждением вблизи дна зоны проводимости фотопроводящего слоя.

В настоящей работе объединены сразу несколько подходов для повышения эффективности антенны: изготовление узкозонных ФСГ InGaAs/InAlAs со сверхмалыми временами жизни возбужденных носителей заряда в фотопроводящих слоях InGaAs, изготовление на основе ФСГ ФПА-источников излучения с плазмонными электродами высокого аспектного соотношения и возбуждение узкозонного материала видимым излучением. По результатам сравнительных измерений средних фототоков, интегральных мощностей $\mathrm{THz}$ излучения и эффективностей оптико-THz преобразования (при разных значениях средней мощности лазерной накачки и приложенного напряжения смещения) нами были определены режимы, в которых ФПА с традиционной топологией могут составлять конкуренцию ФПА с метаповерхностью (плазмонной решеткой).

\section{Образцы и методы исследования}

Используемые в сравнительных экспериментах образцы ФПА с традиционной топологией типа „галстукбабочка“ (bow-tie) и „галстук-бабочка“ с металлической метаповерхностью (плазмонной решеткой) на основе ФСГ InGaAs/InAlAs со сверхмалыми временами жизни фотовозбужденных носителей заряда были спроектированы и изготовлены в ИСВЧПЭ РАН. Зазор электродов для ФПА обоих типов составлял $10 \mu \mathrm{m}$. Узкозонные ФСГ, содержащие тридцатипериодную сверхрешетку $\mathrm{In}_{0.53} \mathrm{Ga}_{0.47} \mathrm{As} / \mathrm{In}_{0.52} \mathrm{Al}_{0.48} \mathrm{As}$, формировались на подложках GaAs методом молекулярно-пучковой эпитаксии со ступенчатым метаморфным буфером [40]. Перед нанесением электродов поверхность ФСГ была покрыта слоем $\mathrm{Si}_{3} \mathrm{~N}_{4}$ толщиной $230 \mathrm{~nm}$, в котором методами оптической фотолитографии вскрывались окна для электрического контакта электродов антенны с поверхностью ФСГ. Топология электродов была сформирована методами фотолитографии со „взрывом“ (lift-off) напыленной системы металлов $\mathrm{Ti} / \mathrm{Au}(50 \mathrm{~nm} / 450 \mathrm{~nm})$. Для создания метаповерхности методом электронно-лучевой литографии со „взрывом“ системы металлов Тi/Au $(18 \mathrm{~nm} / 82 \mathrm{~nm})$ формировалась металлическая решетка: высота одного 
электрода, ширина и зазор между двумя соседними электродами составили $100 \mathrm{~nm}$ (аспектное соотношение между высотой электрода и периодом составляет 1:2). В конце, на поверхность готовой ФПА наносился просветляющий слой $\mathrm{Al}_{2} \mathrm{O}_{3}$ толщиной $180 \mathrm{~nm}$. Технология изготовления образцов антенн подробно описана в работах [41-44].

Регистрация спектров излучения образцов ФПА при разных значениях средней мощности лазерной накачки и приложенного напряжения смещения проводилась с помощью оригинального $\mathrm{THz}$ импульсного спектрометра во временной области $[44,45]$. В качестве источника излучения был использован исследуемый образец ФПА, а в качестве приемника - коммерческий ФПАприемник TERRA-8 (Menlosystems) типа „обвернутый диполь“ (wrapped dipole). На ФПА-источник излучения подавалось переменное смещение $U_{b}$ с возможностью регулировки амплитуды в диапазоне 5-33 V и постоянной частотой следования прямоугольных импульсов $10 \mathrm{kHz}$. Токовый сигнал с ФПА-приемника TERRA-8 после предварительного усиления синхронно выпрямлялся на данной частоте, что позволило обеспечить высокое отношение сигнал/шум $\sim 80 \mathrm{~dB}$. Для накачки обоих ФПА использовались ультракороткие импульсы волоконного лазера EFOA-SH (Авеста-Проект) со следующими параметрами: центральная длина волны $780 \mathrm{~nm}$, частота повторения импульсов $f_{L}=65 \mathrm{MHz}$, длительность импульсов $\sim 85 \mathrm{fs}$. Средняя мощность лазерного излучения в пучке зондирования фиксировалась на уровне $\sim 10 \mathrm{~mW}$. В пучке накачки с помощью регулируемого ослабителя средняя мощность варьировалась в пределах $P_{\text {opt }}=0.1-10.0 \mathrm{~mW}$. При накачке антенн с метаповерхностью вектор поляризации световой волны ориентировался перпендикулярно электродам решетки. Для согласования ФПА-источника и ФПА-приемника излучения со свободным пространством были использованы гиперполусферические линзы из высокорезистивного кремния (high-resistivityfloat-zonesilicon - HRFZ-Si) диаметром $10 \mathrm{~mm}$ и высотой $6.7 \mathrm{~mm}$; сбор и транспортировка $\mathrm{THz}$ излучения осуществлялась внеосевыми параболическими зеркалами диаметром 2 inchs и фокусным расстоянием 4 inchs. Время регистрации спектра $\mathrm{THz}$ излучения одного образца ФПА с частотным разрешением $\sim 0.02 \mathrm{THz}$ для спектрометра составляет $\sim 30 \mathrm{~s}$, что достигается, в том числе, и за счет использования в линии оптической задержки быстродействующей $150 \mathrm{~mm}$ подвижки с линейным приводом, снабженным обратной связью по положению каретки (по сигналам оптического энкодера).

Средние фототоки для исследуемых образцов ФПА $i_{\text {ph }}$ измерялись в диапазоне напряжений $U_{b}=1-70 \mathrm{~V}$ микроамперметром, включенным в цепь питания антенн. Измерения интегральной мощности $\mathrm{THz}$ излучения $P_{\mathrm{THz}}$ выполнялись с помощью абсолютно-калиброванной ячейки Голея, устанавливаемой в непосредственной близости от согласующей $\mathrm{Si}$ линзы исследуемого образца ФПА-излучателя. В силу невысокого быстродействия ячейки Голея, в данных измерениях лазерное излучение в пучке накачки модулировалось механическим прерывателем на частоте $23 \mathrm{~Hz}$. Эффективности оптико-THz преобразования в образцах ФПАисточников излучения были вычислены по результатам измерения интегральных мощностей излучения согласно выражению $\eta=P_{\mathrm{THz}} / P_{\text {opt }}$.

\section{Результаты исследований и их обсуждение}

Спектры генерации исследуемых образцов антенн приведены на рис. 1. Было обнаружено, что при одинаковом напряжении смещения сигналы от образцов ФПА с традиционной топологией существенно слабее, поэтому для регистрации их спектров использовались напряжения $U_{b} \geq 20 \mathrm{~B}$. Видно, что спектральная плотность излучения для ФПА с метаповерхностью и для ФПА с традиционной топологией распределена в широком диапазоне частот и превышает уровень шумов спектрометра вплоть до 2.5-3.0 THz. Особенностью ФПА с метаповерхностью является значительный прирост мощности в низкочастотной области спектра до $0.5 \mathrm{THz}$. Следует отметить, что ФПА с традиционной топологией демонстрируют спад высокочастотного края спектра при снижении средней мощности лазерного излучения, в то время как для ФПА с метаповерхностью форма спектров не зависит ни от приложенного напряжения смещения, ни от средней мощности лазерного излучения.

Типичные зависимости среднего фототока от приложенного к антенне напряжения смещения для образцов ФПА-источников излучения с традиционной топологией и с метаповерхностью приведены на рис. 2, $a$. Видно, что несмотря на малое значение ширины запрещенной зоны $\sim 0.9 \mathrm{eV}$ для слоев $\mathrm{In}_{0.53} \mathrm{Ga}_{0.47} \mathrm{As}$, при традиционной топологии электродов пробой ФПА не наступает вплоть до $U_{b}=60 \mathrm{~V}\left(E_{b}=60 \mathrm{kV} / \mathrm{cm}\right)$, при этом фототок достигает значений $\sim 1 \mathrm{~mA}$. Формирование метаповерхности приводит к тому, что распределение напряженности электрического поля в зазоре становится неоднородным, поэтому пробой антенны даже без лазерной накачки может наступить уже при $U_{b}=30 \mathrm{~V}$.

Выполненные методом оптической накачки-зондирования (pump-probe) измерения показали, что для использованных ФГС при выбранных режимах накачки времена жизни фотовозбужденных носителей заряда составляют $\tau_{c} \sim 5$ ps [40]. За эти времена в ФПА с традиционной топологией достигнуть металлических контактов успевает только небольшая доля фотовозбужденных носителей, пропорциональная средней скорости их дрейфа. В таких образцах в экспериментах наблюдался степенной рост фототока с увеличением напряжения смещения для всех мощностей лазерной накачки (рис. 3, $a$, сплошные линии). В случае наличия метаповерхности фотовозбужденные носители локализованы вблизи контакта металла 

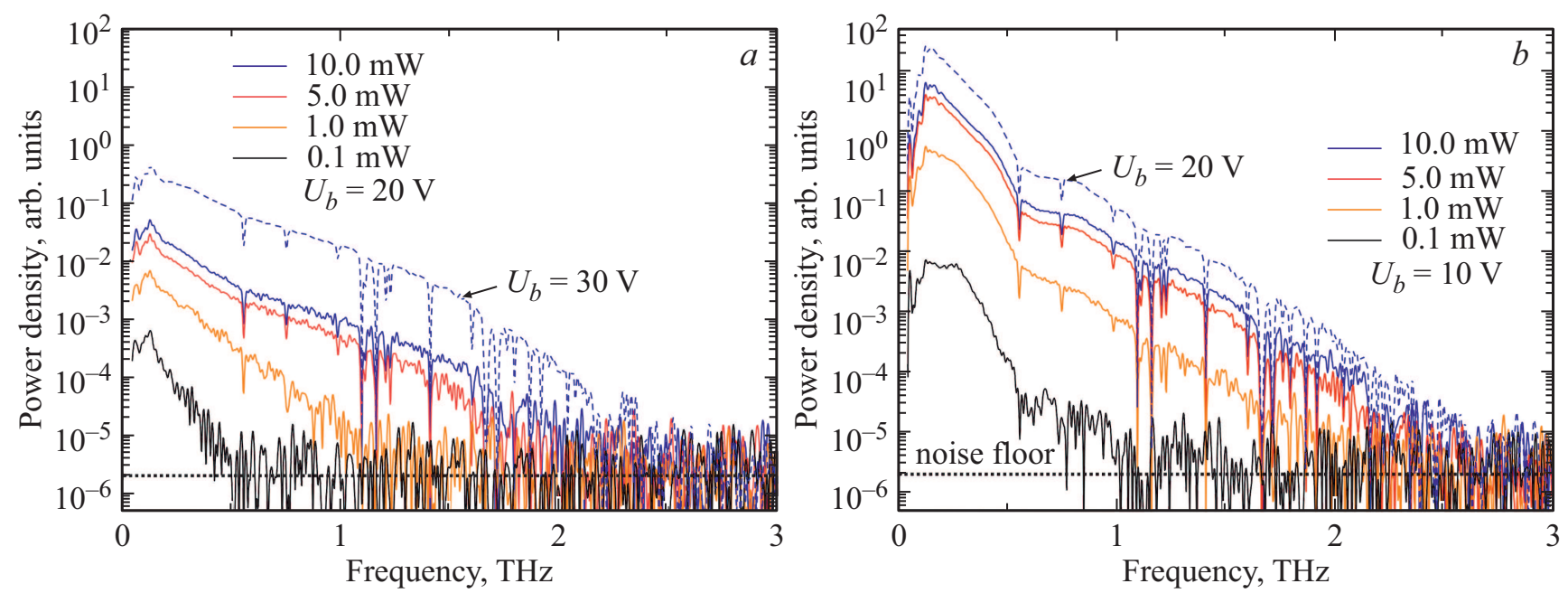

Рис. 1. Спектры излучения при разной средней мощности лазерной накачки для ФПА с традиционной топологией и ФПА с метаповерхностью (плазмонной решеткой) $(a)$ и $(b)$.
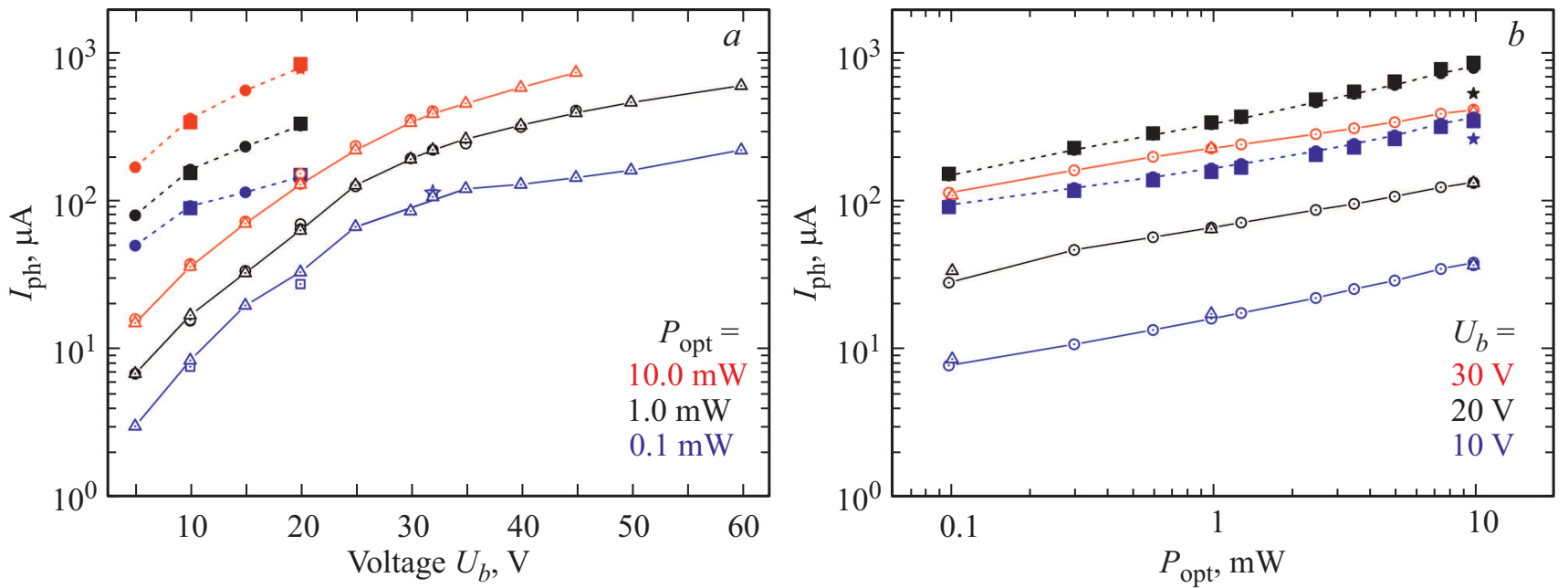

Рис. 2. Фототоки при разных режимах лазерной накачки образцов ФПА: $(a)$ при фиксированной средней мощности лазерной накачки $P_{\text {opt }}$ и $(b)$ при фиксированном напряжении смешения $U_{b}$. Сплошные линии соответствуют ФПА с традиционной топологией, а штриховые линии - ФПА с метаповерхностью (плазмонной решеткой).

решетки с полупроводником, поэтому в экспериментах фототоки мало изменяются с напряжением и быстро достигают значений, характерных для насыщения в традиционной топологии (рис. 2, $a$, штриховые линии). Следует отметить, что для тонких слоев $\operatorname{In}_{0.53} \mathrm{Ga}_{0.47} \mathrm{As}$ зависимость скорости дрейфа носителей от приложенного напряжения будет отличаться от объемного случая, в том числе по причине уменьшения времени релаксации их импульса из-за рассеяния на гетерограницах.

Измеренные зависимости фототоков от средней мощности лазерного излучения показаны на рис. 2, $b$. Для традиционной топологии кривая при $U_{b}=30 \mathrm{~V}$ соответствует случаю, когда при данной накачке максимально возможное число носителей заряда достигает электродов антенны. В случае метаповерхности фотовозбуж- денные носители гораздо легче достигают электродов антенны, и напряжение смещения влияет на величину фототока в меньшей степени, а экспериментальные кривые в целом повторяют зависимость для случая максимального приложенного напряжения в традиционной топологии. Нетрудно показать, что при средней мощности лазерного излучения $P_{\text {opt }} \sim 1 \mathrm{~mW}$ концентрация фотовозбужденных носителей заряда в ФСГ недостаточна для экранирования разделяющимися в пространстве зарядами приложенного к зазору антенны электрического поля смещения [44]. Экспериментальные кривые для образцов ФПА обоих типов при $P_{\text {орt }}>1 \mathrm{~mW}$ не изменяют своего характера, поэтому эффектами насыщения сигналов изза высокого уровня оптической накачки можно пренебречь во всем рассмотренном диапазоне мощности накачки. 

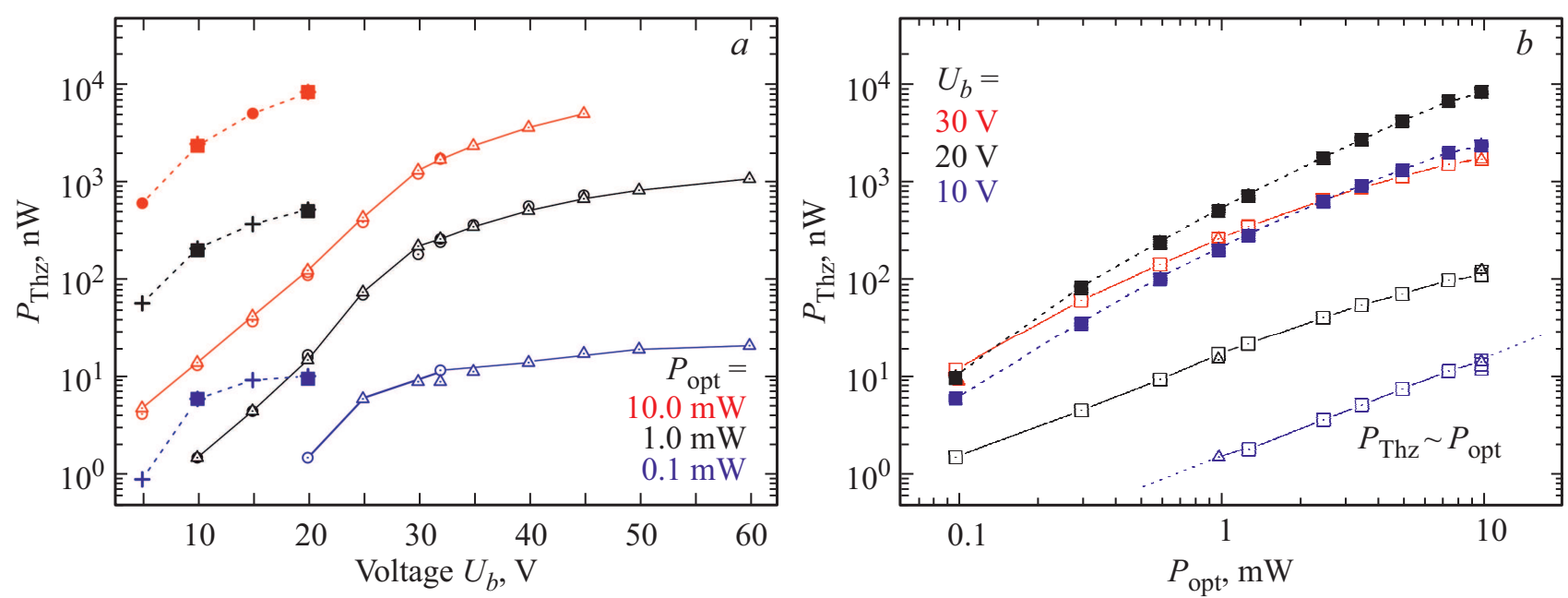

Рис. 3. Интегральная мощность ТНz излучения при разных режимах лазерной накачки образцов ФПА: (a) при постоянной средней мощности лазерной накачки $P_{\text {орt }}$ и $(b)$ при фиксированном напряжении $U_{b}$ смещения. Сплошные линии соответствуют ФПА с традиционной топологией, а штриховые линии - ФПА с метаповерхностью (плазмонной решеткой).
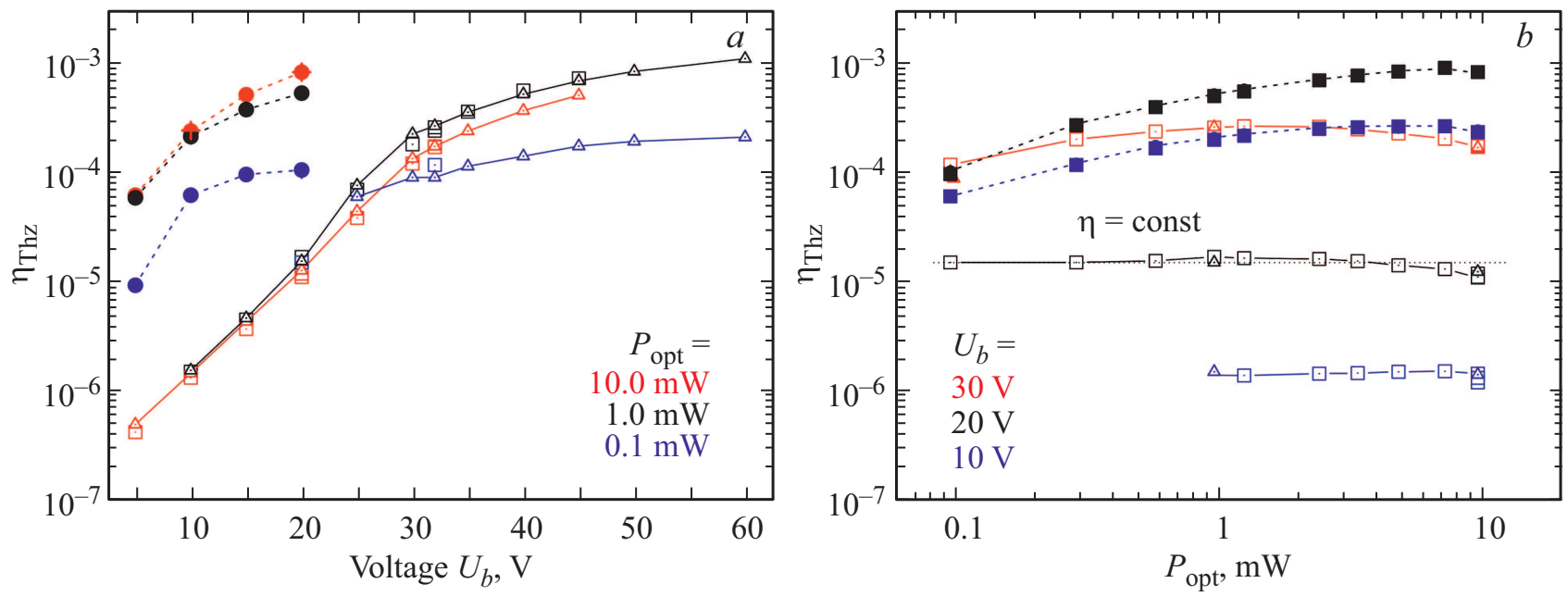

Pис. 4. Эффективности преобразования энергии лазерной накачки в электромагнитные колебания ТНz диапазона при разных режимах лазерной накачки образцов ФПА: $(a)$ при постоянной средней мощности лазерной накачки $P_{\text {орt }}$ и $(b)$ при фиксированном напряжении $U_{b}$ смещения. Сплошные линии соответствуют ФПА с традиционной топологией, а штриховые линии - ФПА с метаповерхностью (плазмонной решеткой).

Зависимости интегральных мощностей $P_{\mathrm{THz}} \mathrm{THz}$ излучения ФПА и эффективностей оптико-THz преобразования в антеннах $\eta$ от приложенного напряжения смещения и мощности накачки приведены на рис. 3 и 4 соответственно. В работах $[46,47]$ показано, что максимальная интегральная мощность $\mathrm{THz}$ излучения ФПА ограничена запасенной в зазоре антенны энергией. Для оценок, пренебрегая влиянием согласования импедансов фотопроводника и антенны на передачу мощности в антенну [44], можно считать $P_{\max } \sim\left(U_{b}^{2} / R_{a}\right) f_{L} \tau_{\mathrm{THz}}$, где полная длительность $\mathrm{THz}$ сигнала $\tau_{\mathrm{THz}} \sim 3 R_{a} C_{a} \sim 1 \mathrm{ps}$, a $R_{a}$ - действительная часть импеданса антенны для частот до $\sim 1.5-2.0 \mathrm{THz}, C_{a}-$ емкость зазоpa антенны. Соответственно, для типичных значений
$U_{b}=10 \mathrm{~V}$ и $R_{a}=70 \Omega$ (для антенны типа „галстукбабочка“ [48]) величина $P_{\max } \sim 400 \mu \mathrm{W}$. Зарегистрированные во всех экспериментах величины интегральной мощности не превосходят $10 \mu \mathrm{W}\left(P_{\mathrm{THz}} \ll P_{\max }\right)$, т.е. несмотря на значительность величины среднего фототока $\sim 1 \mathrm{~mA}$, измерения проводятся в режиме слабой лазерной накачки. В этом случае сложный характер зависимости $P_{\mathrm{THz}}\left(U_{b}\right)$ не связан с преодолением экранирующего действия фотовозбужденных носителей тока, а вероятно определяется зависимостью дрейфовой скорости данных носителей от приложенного напряжения смещения в узкозонном материале ФСГ $\left(\mathrm{In}_{0.53} \mathrm{Ga}_{0.47} \mathrm{As}\right)$. 
Сравнивая результаты $\mathrm{THz}$ измерений для ФПА с традиционной топологией и с метаповерхностью, приведенных на рис. $4, a$, можно отметить, что при одинаковом приложенном напряжении эффективность и интегральная мощность излучения антенны с метаповерхностью оказывается всегда больше, хотя меньшую эффективность преобразования в традиционной антенне можно в некоторой степени скомпенсировать увеличением напряжения. Электрический пробой объемного $\mathrm{In}_{0.53} \mathrm{Ga}_{0.47} \mathrm{As}$ наступает при напряженностях поля $\sim 200 \mathrm{kV} / \mathrm{cm}$ [49] $\left(U_{b}=200 \mathrm{~V}\right)$, однако на практике достичь даже половины этого значения не удается. Вклад тока утечки (темнового тока) в общий ток антенны быстро нарастает и при $U_{b}=60 \mathrm{~V}$ для исследуемых образцов достигает значений $\sim 230 \mu \mathrm{A}$ (при фототоке $\sim 1 \mathrm{~mA}$ ), что резко укорачивает срок службы ФПА и в дальнейшем приводит к тепловому пробою антенны.

Из рис. 4, $b$ видно, что для ФПА с традиционной топологией $P_{\mathrm{THz}} \sim P_{\mathrm{opt}}^{n} \sim P_{\text {opt. }}$ В работах $[46,47]$ снижение показателя $n$ степенной зависимости интегральной мощности $\mathrm{THz}$ от мощности накачки (в нашем случае до $n=1)$ по сравнению с ожидаемым из общих соображений значением $n=2$ авторы связывают с эффектами насыщения. В случае ФСГ, вероятно, играет роль насыщение ловушечных состояний (центров захвата), обеспечивающих ультракороткие времена жизни фотовозбужденных носителей в $\operatorname{In}_{0.53} \mathrm{Ga}_{0.47} \mathrm{As}$, что связано с меньшей плотностью центров захвата по сравнению со случаями формирования таких же центров за счет ионной имплантации или компенсирующего легирования [22-26]. Отметим, что данное замечание также справедливо и в отношении слабой зависимости среднего фототока от мощности накачки.

Вследствие низкого показателя степени $n$ компенсировать невысокую интегральную мощность $\mathrm{THz}$ излучения за счет увеличения мощности лазерной накачки нецелесообразно в том числе из-за перехода в режим сильной накачки, другими словами, снижения эффективности оптико-THz преобразования за счет экранирования электрического поля. Скорее всего, именно экранированием внешнего электрического поля объясняется характер зависимости излучаемой $\mathrm{THz}$ мощности от лазерной накачки для $U_{b}=30 \mathrm{~V}$ уже при $P_{\text {opt }}>5 \mathrm{~mW}$ в антенне с традиционной топологией (рис. $3, b$ ). Таким образом, если для решения прикладных задач медицинской диагностики требуются значения интегральной мощности $\mathrm{THz}$ излучения на уровне $\sim 5-10 \mu \mathrm{W}$, то в качестве источников излучения следует использовать ФПА с метаповерхностью. При уровнях мощности $\sim 1-5 \mu \mathrm{W}$ и менее преимущества ФПА с метаповерхностью по сравнению ФПА с традиционной топологией становятся несущественными.

В результате было обнаружено, что эффективность оптико-THz преобразования для ФПА с традиционной топологией не превосходит $\eta=0.1 \%$ (для $U_{b}=50 \mathrm{~V}$ ). Из сравнения значений $\eta$ на рис. 4, $a$ можно сделать вывод, что ФПА с традиционной топологией могут конку- рировать с ФПА с метаповерхностью, но только работая в режиме, близком к тепловому пробою антенны. С другой стороны, эффективность преобразования в ФПА с метаповерхностью имеет тенденцию к последующему увеличению и с напряжением, и с мощностью накачки. В частности, при $U_{b}=30 \mathrm{~V}$ и $P_{\text {opt }}=3.4 \mathrm{~mW}$ нам удалось достичь $\eta=0.2 \%$ при относительно безопасном для антенны фототоке $I_{\mathrm{ph}}=0.9 \mathrm{~mA}$.

\section{Заключение}

В настоящей работе рассматривалось несколько подходов к повышению эффективности фотопроводящих антенн (ФПА) - источников излучения: изготовление фотопроводящих сверхрешеточных гетероструктур InGaAs/InAlAs со сверхмалыми временами жизни фотовозбужденных носителей заряда, изготовление на основе этих гетероструктур ФПА с традиционной топологией и с метаповерхностью (плазмонными решетками с высоким аспектным соотношением) и возбуждение узкозонного материала видимым излучением.

Для образцов ФПА были измерены средние фототоки, интегральные мощности $\mathrm{THz}$ излучения и эффективности преобразования энергии лазерного излучения в электромагнитные колебания $\mathrm{THz}$ диапазона при разных средней мощности лазерной накачки и приложенном напряжении смещения. Использование метаповерхности позволило достичь значений интегральной мощности $\mathrm{THz}$ излучения $\sim 10 \mu \mathrm{W}$ при напряжении $20 \mathrm{~V}$ и средней мощности лазерной накачки $10 \mathrm{~mW}$. Максимальная эффективность преобразования составила $\eta=0.2 \%$, что недостижимо для ФПА с традиционной топологией вследствие их теплового пробоя. Главным образом это связано с различием в спектрах излучения антенн для частот менее $0.5 \mathrm{THz}$. Сравнительный анализ показал, что для решения прикладных задач, где требуется работать с интегральной мощностью $\mathrm{THz}$ излучения на уровне $\sim 5-10 \mathrm{~W}$, в качестве источников излучения следует использовать ФПА с метаповерхностью, а для уровня мощности $\sim 1-5 \mu \mathrm{W}$ и менее преимущества ФПА с метаповерхностью по сравнению с ФПА с традиционной топологией становятся несущественными.

\section{Финансирование работы}

Работа была выполнена при финансовой поддержке гранта РНФ 19-79-10240. Изготовление ФСГ проводилось при финансовой поддержке гранта Президента МК5450.2018.2.

\section{Конфликт интересов}

Авторы заявляют, что у них нет конфликта интересов. 


\section{Список литературы}

[1] Zaytsev K.I., Dolganova I.N., Chernomyrdin N.V, Katyba G.M., Gavdush A.A., Cherkasova O.P., Komandin G., Shchedrina M.A., Khodan A.N., Ponomarev D.S., Reshetov I.V., Karasik V., Skorobogatiy M., Kurlov V.N., Tuchin V.V. // J. Optics. 2019. V. 22. N 1. P. 013001.

[2] Sun Q., He Y., Liu K., Fan S., Parrott E.P.J, PickwellMacPherson E. // Quantitative Imagingin Medicine and Surgery. 2017. V. 7. P. 345.

[3] Yang X., Zhao X., Yang K., Liu Y., Liu Y., Fu W., Luo Y. // Trends in Biotechnology. 2016. V. 34. N 10. P. 810.

[4] Smolyanskaya O.A., Chernomyrdin N.V., Konovko A.A., Zaytsev K.I., Ozheredov I.A., Cherkasova O.P., Nazarov M.M., Guilleti J.P., Kozlova S.A., Kistenev Yu.V., Coutaz J.-L., Mounaix P., Vaks V.L., Son J.-H., Cheon H., Wallac V.P., Feldman Yu., Popov N.I., Tuchin V.V. // Progress in Quantum Electronics. 2018. V. 62. P. 1.

[5] Woodward R.M., Wallace V.P., Pye R.J., Cole B.E., Arnone D.D., Linfield E.H., Pepper M. // J. Investigative Dermatology. 2003. V. 120. N 1. P. 72.

[6] Wallace V.P., Fitzgerald A.J., Shankar S., Flanagan N., Pye R., Cluff J., Arnone D.D. // British J. Dermatology. 2004. V. 151. N 2. P. 424.

[7] Joseph C.S., Patel R., Neel V.A., Giles R.H., Yaroslavsky A.N. // J. Biophotonics. 2014. V. 7. N 5. P. 295.

[8] Zaytsev K.I., Kudrin K.G., Karasik V.E., Reshetov I.V., Yurchenko S.O. // Appl. Phys. Lett. 2015. V. 106. N 5. P. 053702.

[9] Sim Y., Park J.Y., Ahn K.M., Park C., Son J.H. // Biomedical Optics Express. 2013. V. 4. N 8. P. 1413.

[10] Reid C.B., Fitzgerald A., Reese G., Goldin R., Tekkis P., O'Kelly P.S., Pickwell-MacPherson E., Gibson A.P., Wallace V.P. // Physics in Medicine \& Biology. 2011. V. 56. N 14. P. 4333.

[11] Doradla P., Alavi K., Joseph C.S., Giles R.H. // J. Biomedical Optics. 2013. V. 18. N 9. P. 090504.

[12] Hou D., Li X., Cai J., Ma Y., Kang X., Huang P., Zhang G. // Physics Medicine \& Biology. 2014. V. 59. N 18. P. 5423.

[13] Fitzgerald A.J., Wallace V.P., Jimenez-Linan M., Bobrow L., Pye R.J., Purushotham A.D., Arnone D.D. // Radiology. 2006. V. 239. N 2. P. 533.

[14] Ashworth P.C., Pickwell-MacPherson E., Provenzano E., Pinder S.E., Purushotham A.D., Pepper M., Wallace V.P. // Optics Express. 2009. V. 17. N 15. P. 12444.

[15] Truong B.C.Q., Tuan H.D., Fitzgerald A.J., Wallace V.P., Nguyen H.T. // IEEE Transactions on Biomedical Engineering. 2015. V. 62. N 2. P. 699.

[16] Oh S., Kim S.H., Ji Y.B., Jeong K., Park Y., Yang J., Park D.W., Noh S.K., Kang S.G., Huh Y.M., Son J.H., Suh J.S. // Biomedical Optics Express. 2014. V. 5. N 8. P. 2837.

[17] Ji Y., Oh S.J., Kang S.G., Heo J., Kim S.H., Choi Y., Song S., Son H.Y., Kim S.H., Lee J.H., Haam S.J., Huh Y.M., Chang J.H., Joo C., Suh J.S. // Scientific Reports. 2016. V. 6. P. 36040.

[18] Yamaguchi S., Fukushi Y., Kubota O., Itsuji T., Ouchi T., Yamamoto S. // Scientific Reports. 2016. V. 6. P. 30124.

[19] Gavdush A.A., Chernomyrdin N.V., Malakhov K.M., Beshplav S.-I.T., Dolganova I.N., Kosyrkova A.V., Nikitin P.V., Musina G.R., Katyba G.M., Reshetov I.V., Cherkasova O.P., Komandin G.A., Karasik V.E., Potapov A.A., Tuchin V.V., Zaytsev K.I. // J. Biomedical Optics. 2019. V. 24. N 2. P. 027001. doi 1.JBO.24.2.027001
[20] Lee Y.S. Principles of Terahertz Science and Technology. Springer, 2009.

[21] Yahyapour M., Jahn A., Dutzi K., Puppe T., Leisching P., Schmauss B., Vieweg N., Deninger A. // Appl. Sci. 2019. V. 9. P. 1283. doi 10.3390/app9071283

[22] Huber R., Brodschelm A., Tauser F., Leitenstorfer A. // Appl. Phys. Lett. 2000. V. 76. N 22. P. 3191.

[23] Chimot N., Mangeney J., Mounaix P., Tondusson M., Blary K., Lampin J.F. // Appl. Phys. Lett. 2006. V. 89. P. 083519.

[24] Suzuki M., Tonouchi M. // Appl. Phys. Lett. 2005. V. 86. P. 163504. doi 10.1063/1.1901817

[25] Globisch B., Dietz R.J.B., Kohlhaas R.B., Göbel T., Schell M., Alcer D., Semtsiv M., Masselink W.T. // J. Applied Physics. 2017. V. 121. P. 053102. doi $10.1063 / 1.4975039$

[26] Dietz R.J., Globisch B., Roehle H., Stanze D., Göbel T., Schell M. // Opt. Express. 2014. V. 22 P. 19411.

[27] Ospald F., Maryenko D., Klitzing K., Driscoll D.C., Hanson M.P., Lu H., Gossard A.C., Smet J.H. // Appl. Phys. Lett. 2008. V. 92. P. 131117.

[28] Roehle H., Dietz R.J.B., Hensel H.J., Böttcher J., Künzel H., Stanze D., Schell M., Sartorius B. // Opt. Express. 2010. V. 18. P. 2296.

[29] Ponomarev D.S., Gorodetsky A., Yachmenev A.E., Pushkarev S.S., Khabibullin R.A., Grekhov M.M., Zaytsev K.I., Khusyainov D.I., Buryakov A.M., Mishina E.D. // J. Appl. Phys. 2019. V. 125. N 15. P. 151605.

[30] Glinskiy I.A., Khabibullin R.A., Ponomarev D.S. // Russian Microelectronics. 2017. V. 46. P. 408. doi $10.1134 / \mathrm{S} 1063739717060051$

[31] Khiabani N., Huang Y., Shen Y., Boyes S. // IEEE Transactions on Antennas and Propagation. 2013. V. 61. N 4. P. 1538.

[32] Yachmenev A.E., Lavrukhin D.V., Glinskiy I.A., Zenchenko N.V., Goncharov Y.G., Spektor I.E., Khabibullin R.A., Otsuji T., Ponomarev D.S. // Opt. Eng. 2019. V. 59. N 6. P. 061608. doi 10.1117/1.OE.59.6.061608

[33] Lavrukhin D.V., Yachmenev A.E., Glinskiy I.A., Khabibullin R.A., Goncharov Y.G., Ryzhii M., Otsuji T., Spector I.E., Shur M., Skorobogatiy M., Zaytsev K.I., Ponomarev D.S. // AIP Advances. 2019. V. 9. P. 015112. doi 10.1063/1.5081119

[34] Catrysse P.B., Veronis G., Shin H., Shen J.-T., Fan S. // Appl. Phys. Lett. 2006. V. 88. N P. 031101.

[35] Park S.G., Choi Y., Oh Y.-J., Jeong K.-H. // Optics Express. 2012. V. 20. N 23. P. 25530.

[36] Hsieh B.Y., Jarrahi M. // J. Appl. Phys. 2011. V. 109. P. 084326.

[37] Dietz R., Brahm A., Velauthapillai A., Wilms A., Lammers C., Globisch B., Koch M., Notni G., Schell M. // J. Infrared, Millimeter, Terahertz Waves. 2015. V. 36. N 1. P. 60.

[38] Dietz R.J.B., Wilk R., Globisch B. // J. Infrared Milli. Terahz Waves. 2013. V. 34. P. 231.

[39] Hatem O., Freeman J.R., Cunningham J.E. // J. Infrared Milli. Terahz Waves. 2016. V. 37. P. 415.

[40] Ponomarev D.S., Khabibullin R.A., Klochkov A.N., Yachmenev A.E., Bugaev A.S., Khusyainov D.I., Buriakov A.M., Bilyk V.P., Mishina E.D. // Semiconductors. 2018. V. 52. N 7. P. 864.

[41] Ponomarev D.S., Khabibullin R.A., Yachmenev A.E., Pavlov A.Yu., Slapovskiy D.N., Glinskiy I.A., Lavrukhin D.V., Ruban O.A., Maltsev P.P. // Semiconductors. 2017. V. 51. N 9. P. 1218. 
[42] Lavrukhin D.V., Katyba G.M., Yachmenev A.E., Galiev R.R., Glinskiy I.A., Khabibullin R.A., Goncharov Y.G., Spektor I.E., Khusyainov D.I., Buryakov A.M., Mishina E.D., Chernomyrdin N.V., Zaytsev K.I., Ponomarev D.S. // Proceedings of SPIE. 2018. V. 10680. P. 106801M.

[43] Лаврухин Д.В., Галиев Р.Р., Павлов А.Ю., Ячменев А.Э., Майтама М.В., Глинский И.А., Хабибуллин Р.А., Гончаров Ю.Г., Зайщев К.И., Пономарев Д.С. // Опт. и спектр. 2019. T. 126. B. 5. C. 663.

[44] Lavrukhin D.V., Yachmenev A.E., Pavlov A.Yu., Khabibullin R.A., Goncharov Yu.G., Spektor I.E., Komandin G.A., Yurchenko S.O., Chernomyrdin N.V., Zaytsev K.I., Ponomarev D.S. // Semicond. Sci. Technol. 2019. V. 34. P. 034005 .

[45] Mishina E.D., Grishunin K.A. // Russian Technological J. 2017. V. 5. N 3. P. 41.

[46] Brown E.R., McIntosh K.A., Nichols K.B., Dennis C.L. // Appl. Phys. Lett. 1995. V. 66. P. 285. doi 10.1063/1.113519

[47] Suen J.Y., Li W., Taylor Z.D., Brown E.R. // Appl. Phys. Lett. 2010. V. 96. P. 141103. doi 10.1063/1.337440

[48] Balanis C.A. Antenna Theory: Analysis and Design. 3rd edn. Wiley-Blackwell, 2005. 1073 p.

[49] Hava S., Auslender M. // J. Appl. Phys.1993. V. 73. N 11. P. 7431. 